International Journal of Applied Mathematical Research, 1 (4) (2012) 402-408

\title{
New soliton solutions for Kaup-Boussinesq system
}

\author{
Houshang Najafi, Maliheh Najafi, Somayeh Arbabi Mohammad-Abadi \\ Department of Mathematics, Anar Branch, Islamic Azad University, Anar, Iran \\ Corresponding author: s.arbabi1579@yahoo.com
}

\begin{abstract}
In this Letter, we study Kaup-Boussinesq system by using the wellknown He's variational approach. In fact, the He's variational method is a promising method to various systems of linear and nonlinear equations.
\end{abstract}

Keywords: Variational method, Solitary solution, Kaup-Boussinesq system

\section{Introduction}

Most scientific problems and physical phenomena occur nonlinearly. In fact we can present many important phenomena and dynamic processes in physics, mechanics, chemistry, biology, nonlinear optics, the theory of shallow water waves, plasma physics and others by nonlinear partial differential equations. The study of exact solutions of nonlinear evolution equations plays an important role in soliton theory and explicit formulas of nonlinear partial differential equations play an essential role in the nonlinear science. Also, the explicit formulas may provide physical information and help us to understand the mechanism of related physical models.

Recently, there have been a multitude of methods presented for solving Nonlinear partial differential equations (NPDEs), for instance, the Adomian decomposition method [1],the homotopy perturbation method [2], the variational iteration method [3, 4], the He's variational approach [5], the $F$-expansion method [6], three-wave method [7], extended homoclinic test approach [8, 9], the $\left(\frac{G^{\prime}}{G}\right)$-expansion method [6] and the exp-function method [10]. 
In this paper, by means of the He's variational approach, we will obtain some Solitary solutions of the following Kaup-Boussinesq system given in [11]

$$
\begin{aligned}
& u_{t}-v_{x x x}-2 v u_{x}-2 u v_{x}=0, \\
& v_{t}-u_{x}-2 v v_{x}=0 .
\end{aligned}
$$

\section{He's variational method}

In order to seek its travelling wave solution, we introduce a transformation

$$
u(x, t)=u(\xi) \quad, \quad v(x, t)=v(\xi) \quad, \quad \xi=x-c t
$$

by substituting Eqs. (2) into Eq. (1), we have

$$
\begin{gathered}
-c u^{\prime}-v^{\prime \prime \prime}-2 v u^{\prime}-2 u v^{\prime}=0, \\
-c v^{\prime}-u^{\prime}-2 v v^{\prime}=0 .
\end{gathered}
$$

We can rewrite Eq. (4) in the form

$$
u^{\prime}+c v^{\prime}+2 v v^{\prime}=0
$$

where prime denotes the differential with respect to $\xi$. Integrating Eq. (5) with respect to $\xi$ and taking the integration constant as zero yields

$$
u=-c v-v^{2} .
$$

Now, inserting Eq. (6) into Eq. (3), yields

$$
c^{2} v^{\prime}+6 c v v^{\prime}+6 v^{2} v^{\prime}-v^{\prime \prime \prime}=0 .
$$

Now, Integrating Eq. (7) with respect to $\xi$ and taking the integration constant as zero yields

$$
v^{\prime \prime}-c^{2} v-3 c v^{2}-2 v^{3}=0 .
$$

According to Ref. [12], By He's semi-inverse method [13], we can arrive at the following variational formulation:

$$
J(\phi)=\int_{0}^{\infty}\left[-\frac{1}{2}\left(v^{\prime}\right)^{2}-\frac{\left(c^{2}\right)}{2} v^{2}-c v^{3}-\frac{1}{3} v^{4}\right] d \xi .
$$

We assume the soliton solution in the following form

$$
v(\xi)=A \operatorname{sech}(\xi),
$$


where $A$ is an unknown constant to be further determined.

By Substituting Eq. (10) into Eq. (9) we obtain

$$
J=-\frac{1}{4} A^{3} c \pi-\frac{1}{3} A^{4}-\frac{1}{2} c^{2} A^{2}-\frac{1}{6} A^{2},
$$

For making $J$ stationary with respect to $A$

$$
\frac{\partial J}{\partial A}=-\frac{3}{4} A^{2} c \pi-\frac{4}{3} A^{3}-c^{2} A-\frac{1}{3} A,
$$

From Eq. (12), we have

$$
A=-\frac{9}{32} c \pi+\frac{1}{32} \sqrt{81 c^{2} \pi^{2}-256-768 c^{2}} .
$$

The solitary solutions are, therefore, obtained as follows:

$$
v(\xi)=A \operatorname{sech}(\xi)
$$

and

$$
u(\xi)=-c A \operatorname{sech}(\xi)-A^{2} \operatorname{sech}^{2}(\xi),
$$

where $A=-\frac{9}{32} c \pi+\frac{1}{32} \sqrt{81 c^{2} \pi^{2}-256-768 c^{2}}$.

In this solutions $c$ is an arbitrary real parameter. Fig. 1 and Fig. 2 show the graph of $u(x, t)$ and $v(x, t)$ for $c=\pi,-30 \leq x \leq 30$ and $0 \leq t \leq 5$.

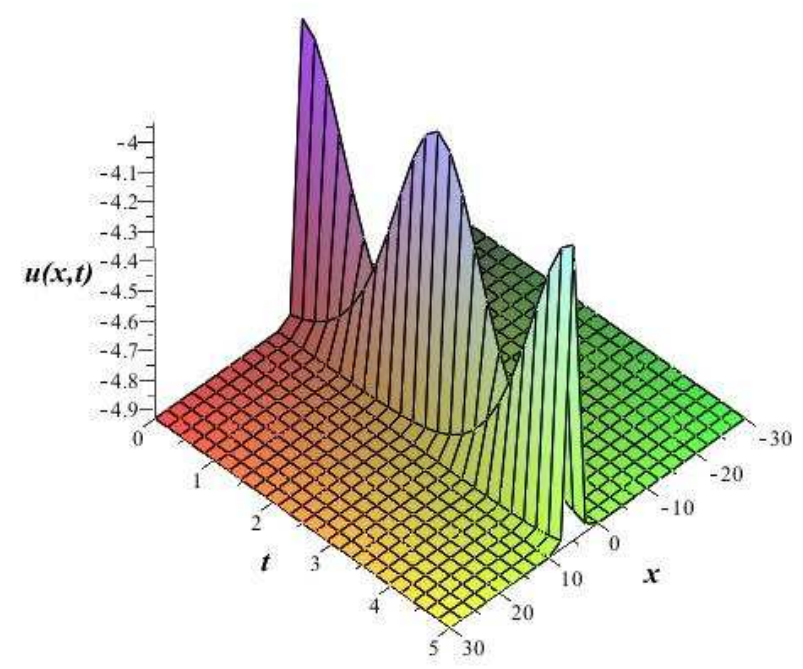

Figure 1: The Soliton solution of Eq. (14). 


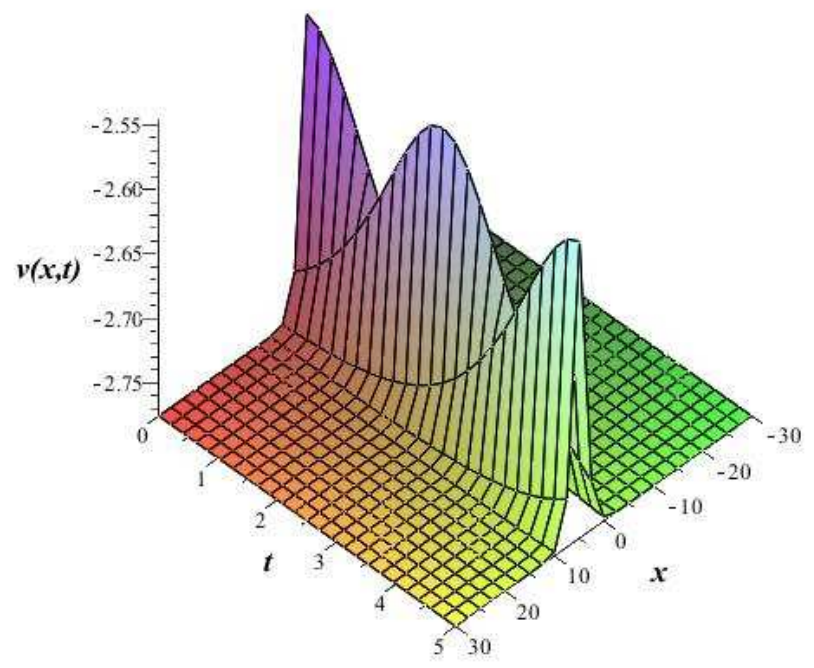

Figure 2: The Soliton solution of Eq. (15).

We search another soliton solution in the form

$$
u(\xi)=D \operatorname{sech}^{2}(\xi),
$$

where $D$ is an unknown constant to be further determined.

By Substituting Eq. (16) into Eq. (9) we obtain

$$
J=-\frac{8}{35} D^{4}-\frac{8}{15} c D^{3}-\frac{1}{3} c^{2} D^{2}-\frac{4}{15} D^{2},
$$

For making $J$ stationary with respect to $D$

$$
\frac{\partial J}{\partial D}=-\frac{32}{35} D^{3}-\frac{8}{5} c D^{2}-\frac{2}{3} c^{2} D-\frac{8}{15} D,
$$

From Eq. (18), we have

$$
D=-\frac{7}{8} c+\frac{1}{24} \sqrt{21 c^{2}-336},
$$

The solitary solutions are, therefore, obtained as follows:

$$
v(\xi)=D \operatorname{sech}^{2}(\xi),
$$

and

$$
u(\xi)=-c D \operatorname{sech}^{2}(\xi)-D^{2} \operatorname{sech}^{4}(\xi),
$$

where $D=-\frac{7}{8} c+\frac{1}{24} \sqrt{21 c^{2}-336}$.

In this solutions $c$ is an arbitrary real parameter. Fig. 3, Fig. 4 show the graph of $u(x, t)$ and $v(x, t)$ for $c=5 \pi,-30 \leq x \leq 30$ and $0 \leq t \leq 5$. 


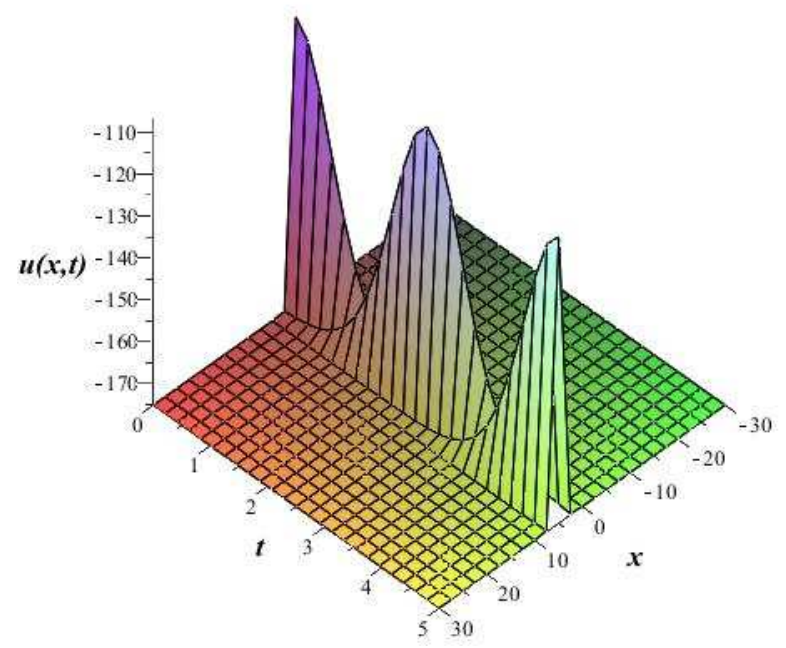

Figure 3: The Soliton solution of Eq. (20).

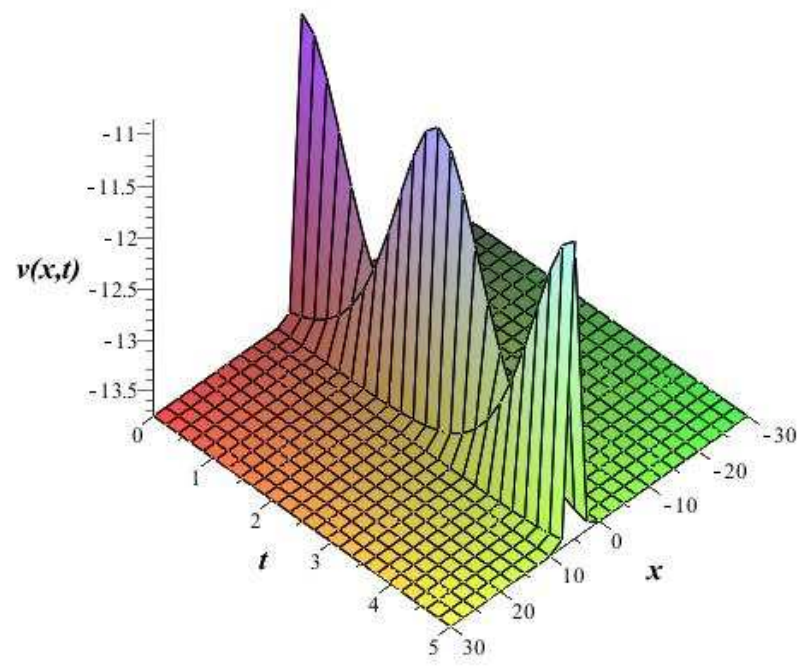

Figure 4: The Soliton solution of Eq. (21).

\section{Conclusion}

In this paper, we used He's variational method to search for solitary solutions of Kaup-Boussinesq system. He's variational principle is a very dominant instrument to find the solitary solutions for various nonlinear equations. 


\section{References}

[1] S. Abbasbandy, M.T. Darvishi, A numerical solution of Burgers equation by modified Adomian method, Appl. Math. Comput. 163 (2005) 12651272 .

[2] J.H. He, Application of homotopy perturbation method to nonlinear wave equations, Chaos, Solitons and Fractals 26(3) (2005) 695-700.

[3] J.H. He, X.H. Wu, Construction of solitary solution and compacton-like solution by variational iteration method, Chaos, Solitons and Fractals, 29 (2006) 108-113.

[4] M.T. Darvishi, F. Khani, S. Hamedi-Nezhad, S.-W. Ryu, New modification of the HPM for numerical solutions of the sine-Gordon and coupled sine-Gordon equations, Int. J. Comput. Math. 87(4) (2010) 908-919.

[5] Y. Khan, N. Faraz, A. Yildirim, New soliton solutions of the generalized Zakharov equations using Hes variational approach, Applied Mathematics Letters., 24 965-968 (2011).

[6] M.T. Darvishi, Maliheh Najafi, Mohammad Najafi, Traveling wave solutions for the $(3+1)$-dimensional breaking soliton equation by $\left(\mathrm{G}^{\prime} / \mathrm{G}\right)$ expansion method and modified F-expansion method, International Journal of Computational and Mathematical Sciences, 6 (2012) 64-69 .

[7] M.T. Darvishi, M. Najafi, M. Najafi, New exact solutions for the $(3+1)$ dimensional breaking soliton equation, International Journal of Engineering and Mathematical Sciences, 6(2) (2010) 137-140.

[8] M.T. Darvishi, Mohammad Najafi, A modification of extended homoclinic test approach to solve the $(3+1)$-dimensional potential-YTSF equation, Chin. Phys. Lett., 28(4) (2011) 040202.

[9] Mohammad Najafi, Maliheh Najafi, M.T. Darvishi, New exact solutions to the $(2+1)$-dimensional Ablowitz-Kaup-Newell-Segur equation: Modication of extended homoclinic test approach, Chin. Phys. Lett., 29(4) (2012) 040202.

[10] J.H. He, M.A. Abdou, New periodic solutions for nonlinear evolution equations using Exp-function method, Chaos, Solitons and Fractals 34 (2007) 1421-1429.

[11] J. Zhou, L. Tian, X. Fan, Solitary-wave solutions to a dual equation of the KaupBoussinesq system, Nonlinear Anal. 11 (2010) 3229-3235. 
[12] J.H. He, Some asymptotic methods for strongly nonlinear equations, Internat. J. Modern Phys. B 20 (10) (2006) 1141-1199.

[13] J.H. He, Variational principles for some nonlinear partial differential equations with variable coefficients, Chaos Solitons Fractals 19 (4) (2004) 847851. 